Noname manuscript No.

(will be inserted by the editor)

\title{
2D Lid-Driven Cavity Flow Simulation using GPU-CUDA with a High Order Finite Difference Scheme
}

\author{
Ediguer E. Franco - Helver M. Barrera - Santiago Laín
}

Received: date / Accepted: date

\begin{abstract}
The high parallelism and low cost of the Graphic Processing Units (GPUs) have attracted the interest of scientists and engineers requiring high computational power with a modest investment. This work explores the use of a GPU in the solution of the 2D Lid Driven Cavity Flow (LDCF) problem using the pressure-velocity formulation for Reynolds numbers up to 10000 and turning to a $4^{\text {th }}$ order finite difference scheme for spatial discretization. Results showed good agreement with those reported in the literature. The solver was implemented in both the CPU and the GPU in order to compare their performance, whereupon the latter was seventy times faster.
\end{abstract}

Keywords Navier-Stokes equations · Lid driven cavity flow $\cdot$ GPU $\cdot$ CUDA

\section{Introduction}

The Compute Unified Device Architecture (CUDA) is a parallel computing technology developed by NVIDIA for graphic processing. This technology includes a programming environment that makes accessible to programmers the computing resources of the graphic devices, allowing the development of general purpose programs. Therefore, the graphic processing units (GPUs) can be used by scientists and engineers to drastically

E. Franco, H. Barrera and S. Laín are with

Fluid Mechanics Research Group

Universidad Autónoma de Occidente

Cll 25 \#115-85, Cali, Colombia

Tel.: +57-2-3188000

Fax: $+57-2-5553911$

E-mail: eefranco@uao.edu.co (E. Franco)

E-mail: embarrera@uao.edu.co (H. Barrera)

E-mail: slain@uao.edu.co (S. Laín) increase their computing power with a modest investment of money. As a consequence, the general-purpose computing on graphic processing units (GPGPU) has arisen as an important field of study.

Many papers about methodologies for the implementation of numerical calculations on GPUs and the solution of engineering problems using these devices have been published in the recent years. Some relevant works are related to the efficient implementation of linear algebra routines [Bell \& Garland, 2008], solution of large linear systems [Courtecuisse \& Alard, ], finite element analysis [Cecka et al., 2000, Markall et al., 2013], FFT calculations [Moreland \& Angel, 2003], computational fluid dynamics (CFD) calculations [Frezzotti et al., 2011, Vanka, 2013,Zheng et al., 2013], among others.

The Computational Fluid Dynamics (CFD) is a research field where parallel computing using graphical processing units (GPUs) has many applications. GPU implementations can be assessed by means of benchmark problems, such as the Lid Driven Cavity Flow (LDCF). In the LDCF, the fluid contained in a cavity is driven by a a moving lid, generating a typical flow pattern. This benchmark problem has been widely used for evaluation of CFD solvers because the solution is known in detail and, depending on the Reynolds Number, the mesh sizes and time steps required, the problem solution is intensive in computational terms.

In the solution of the LDCF, most authors have used the velocity-stream function formulation [Cardoso \& Bicudo, 2009, Erturk et al., 2005,Erturk, 2009] because there are only two unknowns and the continuity equation is automatically satisfied. However, this formulation cannot be easily extended to 3D and the implementation of boundary conditions is not easy. In contrast, the velocity-pressure formulation has three unknowns and the solution algo- 
Franco, Barrera and Laín

rithms are more complex, but this formulation can be easily extended to $3 \mathrm{D}$.

Despite the LDCF being a well documented problem, for it is widely used as example in textbooks and as benchmark in research papers, there is not consensus about the Reynolds number for turbulent transition. In this regards, Erturk et al. consider a vorticity formulation, reaching convergence for Reynolds numbers as high as 21000. However, this author suggests that this simulation can only be a realistic model for Reynolds numbers up to 1000 [Erturk, 2009]. In the work of Cardoso and Bicudo [Cardoso \& Bicudo, 2009], the LDCF problem has been solved in a fine mesh using both CPU and GPU. They claim that non stationary results for Re $<30000$ are consequence of coarse meshes.

In this work, the velocity-pressure formulation was used for the solution of the LDCF problem with Reynolds numbers up to 10000. The solver was implemented using the Finite Difference Method with high order discretization schemes for spatial derivatives. The solution was programmed in Ansi C and CUDA, for execution in the CPU and GPU, respectively. The Results are in agreement with a reference solution obtained by the velocity-stream function formulation. The performance analysis showed the GPU was seventy times faster than CPU using double precision arithmetics.

\section{Flow equations}

The incompressible Navier-Stokes equations in a two dimensional Cartesian frame of reference are used to model the LDCF problem of concern. These are a set of nonlinear, time dependent partial differential equations:

$$
\begin{aligned}
\frac{\partial u}{\partial x}+\frac{\partial v}{\partial y} & =0 \\
\frac{\partial u}{\partial t}+\rho \frac{\partial}{\partial x}\left(u^{2}\right)+\rho \frac{\partial}{\partial y}(u v)+\frac{\partial p}{\partial x} & =\mu\left(\frac{\partial^{2} u}{\partial x^{2}}+\frac{\partial^{2} u}{\partial y^{2}}\right) \\
\frac{\partial v}{\partial t}+\rho \frac{\partial}{\partial y}\left(v^{2}\right)+\rho \frac{\partial}{\partial x}(u v)+\frac{\partial p}{\partial y} & =\mu\left(\frac{\partial^{2} v}{\partial x^{2}}+\frac{\partial^{2} v}{\partial y^{2}}\right)
\end{aligned}
$$

where $u$ and $v$ are the horizontal and vertical components of velocity, respectively, $p$ is the pressure, $\rho$ the density and $\mu$ the viscosity.

Equation (1) is the continuity equation. It establishes the conservation of mass through the domain, restricting the velocity to a divergence free vector field. Equations (2) and (3) establish the conservation of the momentum in the horizontal and vertical directions, where the left hand contains the acceleration and the pressure gradient, and the right hand term is viscous force.

An important feature of the governing equations is the so-called velocity-pressure coupling. In equations
(2) and (3) the pressure gradient plays the role of a momentum source term. This source is not a known quantity: it is the pressure field satisfying the continuity equation and has to be calculated at each temporal step. This work follows an approach proposed by Chorin [Chorin, 1968] based on the observation that the velocity field must be solenoidal (divergence free field) in order to satisfy continuity. The velocity-pressure coupling results in the continuity equation being replaced by the following Poisson equation for the pressure:

$\frac{\partial^{2} p}{\partial x^{2}}+\frac{\partial^{2} p}{\partial y^{2}}=\frac{\partial u^{*}}{\partial x}+\frac{\partial v^{*}}{\partial y}$

where $u^{*}$ and $v^{*}$ are the intermediate velocities obtained from the evaluation of the momentum equations without the pressure gradient term. The final velocity is obtained by subtracting the pressure gradient to the velocity in order to force a solenoidal field.

The initial velocity and pressure fields are set to zero in all directions, except in the north boundary where the horizontal velocity is unitary. Boundary conditions are non-slip condition everywhere for velocity (Dirichlet boundary conditions) and zero gradient normal to all boundaries for pressure (von Neumann boundary conditions).

\section{CUDA Technology}

CUDA is a hardware and software architecture developed by NVIDIA to execute programs in parallel. These programs can be both graphic routines or general purpose programs that can be executed in the CUDA enabled graphic devices and written in diverse languages, such as $\mathrm{C}, \mathrm{C}++$, Fortran, OpenCL, Python, among others.

A CUDA program executes a kernel simultaneously. A kernel is a piece of code susceptible of being executed in parallel. For example, the scalarmatrix multiplication can be performed in parallel because each individual multiplication does not depends on the other ones and, thus, can be executed in a different processor at the same time. Other calculation routines cannot be directly parallelized. Each individual call to a kernel is called a thread, and a thread has an ID, a program counter, registers, per-thread private memory (local memory), inputs, and output results. A block is a set of threads concurrently executed. It shares a per-block memory (shared memory) and can be synchronized. A grid is an array of blocks executing the same kernel and it can read and write data in the global memory and synchronize between dependent kernel calls. Each block has an ID within the grid. Figure 1 shows the CUDA hierarchy of threads, blocks and grids. 


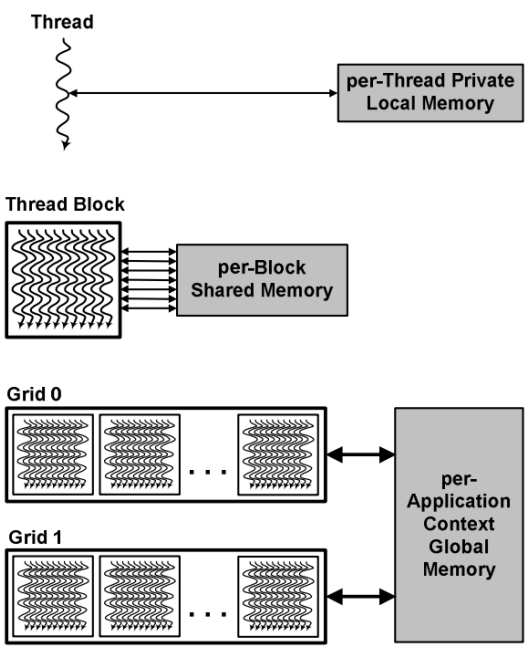

Fig. 1 Hierarchy of CUDA threads, blocks and grids [FER, 2009]

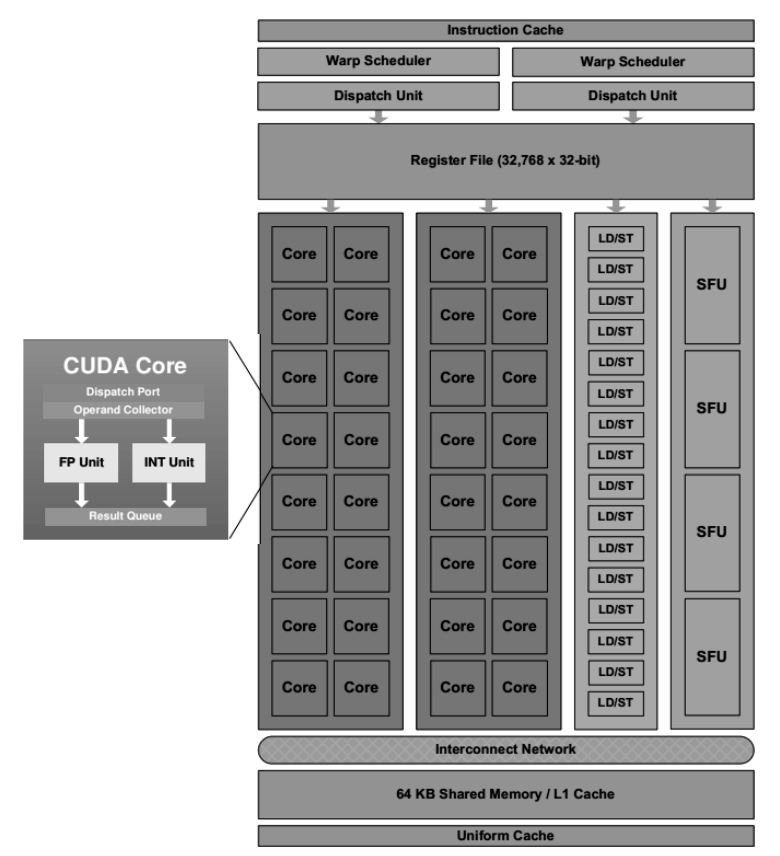

Fig. 2 Scheme of the Fermi architecture [FER, 2009]

Figure 2 shows a scheme of the Fermi streaming processor (SM), featuring 32 cores. Each core has an arithmetic logic unit (ALU) and a floating point unit (FPU). The SM has four special function units (SFUs), which execute transcendental instructions such as sine, cosine, reciprocal, and square root, sixteen load/store units (LD/ST) for addressing, registers, L1 cache and scheduler and dispatch units. Fermi architecture implements the new and more accurate IEEE 754-2008 floating-point standard.

Each device has an array of up to 48 streaming processors (up to 1536 CUDA cores), with a common L2 cache, six partitions of a 64-bit DRAM global mem- ory, a PCI Express host interface for communication with the CPU and a global scheduler that distributes the thread blocks in the streaming processors. All these units work together, interconnected by a network like a computing cluster in a single device.

Software is another important issue. NVIDIA has released a development toolkit for the most important operative systems, including an extensive collection of code samples. These software tools have facilitated the understanding of the technology and its features to general purpose programmers and scientists. A CUDA program is compiled by the NVCC (NVIDIAS's CUDA compiler), available in the development kit. The executable contains the GPU code and CPU routines required to control the operation, i.e., data transfer between CPU and GPU and the kernels scheme of execution.

The CUDA programming is different from the standard programming, because parallelism implies another programing paradigm. Some algorithms can be implemented in GPU almost by translating the CPU code and other ones are difficult to parallelize. Moreover, hardware specific issues, specially the memory management, and the restrictions in the computing features lead to different programming techniques. Information about the architecture and programming can be found in the CUDA documentation and the training resources available in the NVIDIA site.

\section{Numerical implementation}

\subsection{Discretization scheme}

The rectangular domain was discretized using a regular Arakama A-grid. This is a localized mesh, where velocity and pressure values are in the same nodes, causing an instability problem of "checker-board" patterns [Versteeg \& Malalasekera, 1995]. In this work, stability problems normally addressed by staggered grids are partially circumvented using fourth order finite difference schemes for spacial derivatives of $u, v$, and $p$. On typical finite volume approximations the pressure gradient is taken from alternate grid points, so that a wavy pressure can be read as uniform in its role of driving the flow by the momentum equation [Patankar, 1980]. The $4^{\text {th }}$ order finite difference scheme considers up to five grid points for the task, hence the checkerboard pressure pattern is reduced. Table 1 shows the finite difference schemes used in this work.

Regarding the time-marching procedure, time derivative is approximated by first order forward differences. Every time step is composed by two internal sub-steps. The underlying idea is that Navier-Stokes equations 
Table 1 Finite difference schemes used in this work [Chapra \& Canale, 1995]

\begin{tabular}{ll}
\hline Scheme & Error \\
\hline \hline $4^{\text {th }}$ order centered: & \\
$\frac{\partial u}{\partial x} \approx \frac{-u_{i+2}+8 u_{i+1}-8 u_{i-1}+u_{i+2}}{12 \Delta x}$ & $O\left(\Delta x^{4}\right)$ \\
$\frac{\partial^{2} u}{\partial x^{2}} \approx \frac{-u_{i+2}+16 u_{i+1}-30 u_{i}+16 u_{i-1}-u_{i+2}}{12 \Delta x^{2}}$ & $O\left(\Delta x^{4}\right)$ \\
$1^{\text {st }}$ order forward: & \\
$\frac{\partial u}{\partial x} \approx \frac{u_{i+1}-u_{i}}{\Delta x}$ & $O(\Delta x)$ \\
\hline
\end{tabular}

can be solved by separately considering the convective and diffusive terms, and the velocity-pressure coupling [Seibold, 2008]. A temporal step from time $n$ to $n+1$ is implemented in the following way. First, the intermediate velocities $u^{*}$ and $v^{*}$ are calculated by:

$$
\begin{aligned}
& \frac{u^{*}-u^{n}}{\Delta t}=\frac{\mu}{\rho} \nabla^{2} u^{n}-\left(\mathbf{u}^{n} \cdot \nabla\right) u^{n} \\
& \frac{v^{*}-v^{n}}{\Delta t}=\frac{\mu}{\rho} \nabla^{2} v^{n}-\left(\mathbf{u}^{n} \cdot \nabla\right) v^{n}
\end{aligned}
$$

These intermediate velocities are the evaluation of the momentum equations (2) and (3) without the pressure gradient. Next, the pressure field is obtained from the Poisson's equation (4):

$\frac{\partial^{2} p^{n}}{\partial x^{2}}+\frac{\partial^{2} p^{n}}{\partial y^{2}}=\frac{\partial u^{*}}{\partial x}+\frac{\partial v^{*}}{\partial y}$

Finally, the new velocities are obtained by sustracting the pressure gradient to the velocity in order to force a soleniodal velocity field:

$\frac{u^{n+1}-u^{*}}{\Delta t}=-\frac{\partial p^{n}}{\partial x}$

$\frac{v^{n+1}-v^{*}}{\Delta t}=-\frac{\partial p^{n}}{\partial y}$

\subsection{GPU implementation}

The domain is a rectangular array of nodes. The explicit finite difference scheme employed allows the independent calculation at each node. Therefore, there are different possibilities for the parallelization scheme. A group of nodes can be handled by a block of threads, where each thread evaluates the finite difference stencil on the corresponding node. The threads of a block are processed in the same streaming processor (SM) and the parallelization depends on the number of cores in the SM. The GPU architecture have a limit in the number of threads-per-block. In this work, a NVIDIA GeForce GTX-570 card with up to 1024 threads per block was used. However, a higher threads-per-block number does not imply, in general, a better performance. Different blocks can be processed in different SMs at the same time, then, parallelization of blocks depends on the number of the SMs in the GPU. The domain was discretized using a mesh with a number of nodes (Nodes) between $256^{2}$ and $4096^{2}$. The threads per block number (Threads) was fixed to a value between 32 and 512. Then, the number of block was Blocks $=$ Nodes/Threads.

The solution was implemented using only the global memory. In this approach, each thread has to read the values required for the finite difference stencil evaluation from the slower global memory. The surrounding values of each node have to be loaded by the corresponding thread. As a consequence, each node is read multiple times, affecting the performance. The solution of this problem is an approach called data reuse, where a dataset is loaded in the faster shared memory and a group of nodes is evaluated. The bigger dataset, the fewer multiple readings occur and the performance is thereby increased.

For the implementation, initial conditions were set at the beginning in the host memory and then transferred to the device memory where the kernels are dispatched. There are kernels for the operations required by the finite difference scheme at each node, corresponding to the terms given by the original mathematical model: first and second spatial derivatives for vertical and horizontal components of velocity and for pressure.

Calculation data were stored on the device memory as they were produced, only to be released to the host memory after a predefined number of time steps. This allows the monitoring of the time evolution of the variables and to capture a complete set of data to visualize the transient behavior. Post-processing was carried out using specially developed Matlab scripts.

\section{Results and discussion}

The lid driven cavity problem was solved for Reynolds number of 400, 1000, 5000 and 10000 (from laminar to turbulent regimes). The size of the cavity is $L=1$ and the Reynolds number was set by fixing the physical properties of the fluid and the lid velocity. The convergence criterion used to finish the calculation was the maximum difference in the horizontal velocity between two successive iterations be less than a certain tolerance. In order to assure stability of the explicit solver, the time increment $(\Delta t)$ was chosen to have a Courant number of 0.16 . 

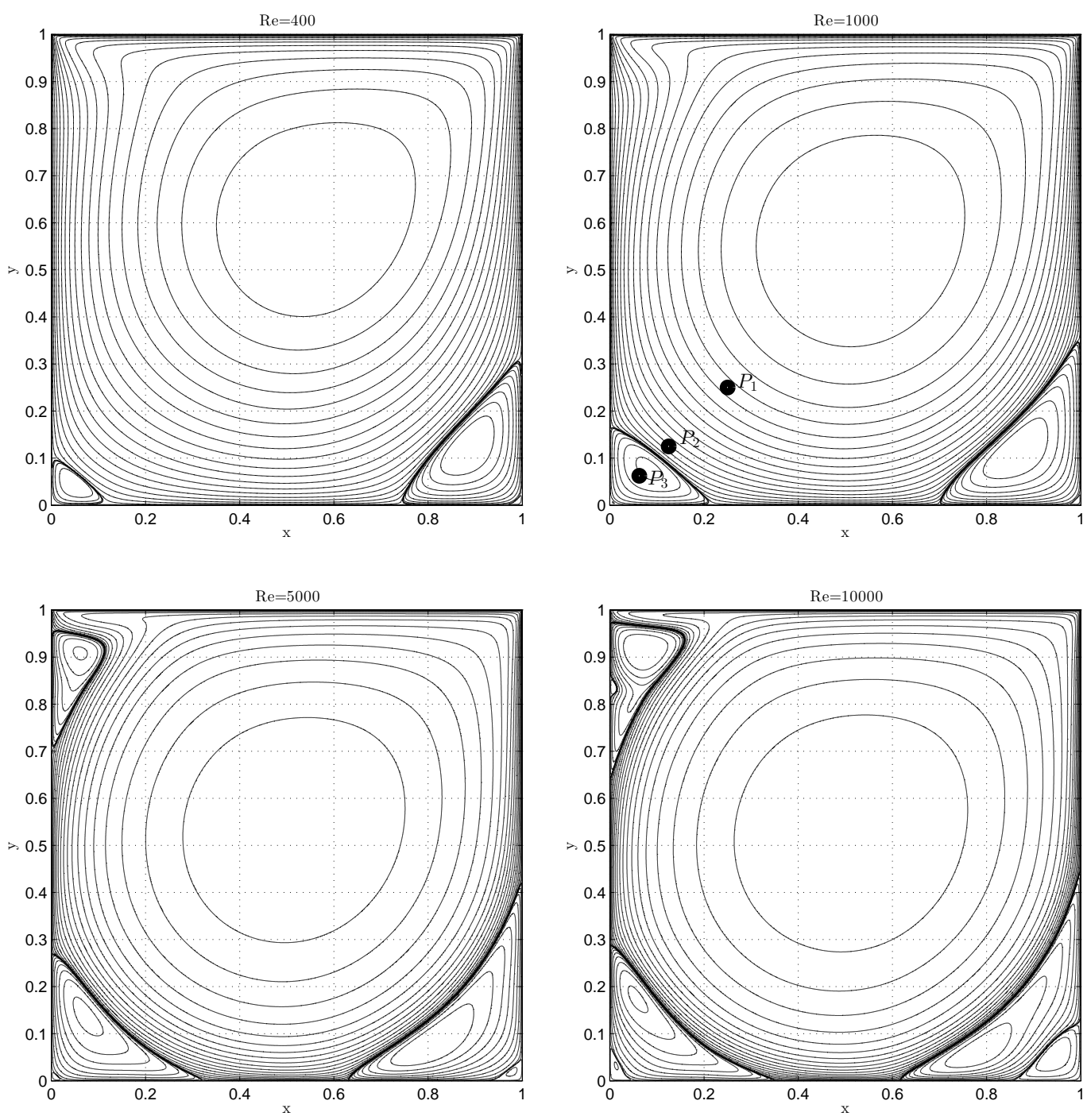

Fig. 3 Streamlines calculated from the velocity vector fields, with discretizations of $1024^{2}$ nodes for $\operatorname{Re}=400,1000$ and 5000 , and $2048^{2}$ nodes for $\operatorname{Re}=10000$.

Figure 3 shows the streamlines for a discretization of $1024^{2}$ nodes, for Reynolds numbers 400, 1000 and 5000, and $2048^{2}$ for $\mathrm{Re}=10000$. Taking into account that the variables are $u, v$ and $p$, there are three millions of degrees of freedom in the first case and sixteen millions in the second one. Velocity and pressure were calculated in a temporal advance scheme until reaching steady state. Then, the stream function was calculated from the velocity vector field in a post-processing step also carried out in the GPU. It can be seen a central (primary) vortex covering a large portion of the cavity and small secondary vortices in the corners. When the Reynolds number increases, the primary vortex moves toward the center of the cavity and the secondary vortices grow. These results are in agreement with other results published in literature [Ghia et al., 1982, Erturk, 2009].
Figure 4 shows a comparison of the velocity patterns in the center of the cavity. For the lowest Reynolds Number, the velocity pattern has a parabolic-like behavior, similar to the Stokes flow solution (pure viscous flow). When the Reynolds number increases, the change of velocity occurs in a smaller region next to the wall and a linear velocity variation is observed in the interior of the domain. These results are compared to the reference solution [Ghia et al., 1982], showing a good agreement in all the Reynolds numbers analyzed. It can be established that the developed GPU code provides results that quantitatively reproduce the ones reported in literature.

Table 2 shows a comparison of the position of the secondary vortices for Reynolds numbers of 1000 and 5000. Results obtained in this work were compared to 

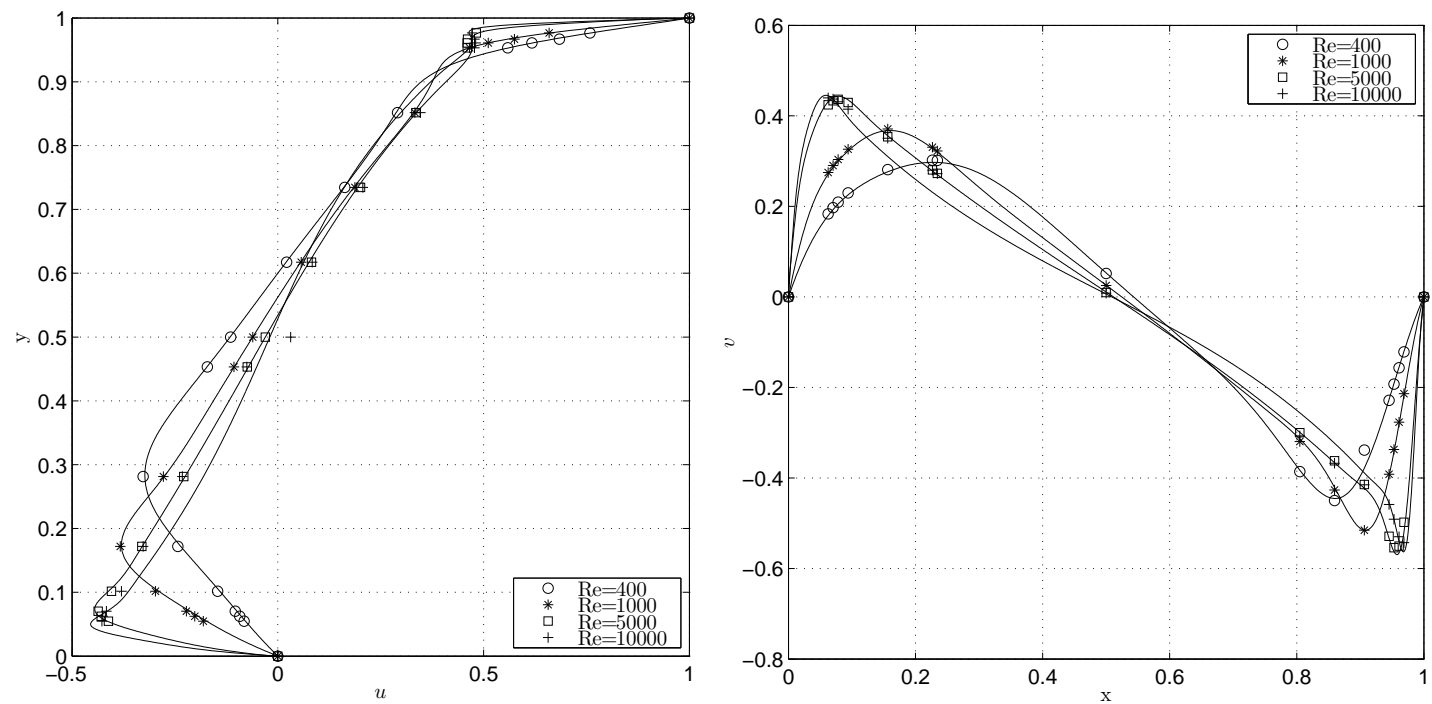

Fig. 4 Horizontal $(u)$ and vertical $(v)$ normalized components of velocity: comparison of the velocity patterns in the center of the domain obtained in this work (solid lines) and obtained by Ghia et al. [Ghia et al., 1982] (markers).

Table 2 Results of the secondary vortices position $(x, y)$ for two Reynolds numbers and comparison with the reference solution (BL: bottom left, BR: bottom right and TL: top left).

\begin{tabular}{lrc}
\hline Vortex & This work & Erturk 2009 [Erturk, 2009] \\
\hline \hline \multicolumn{2}{c}{ Re $=1000:$} & \\
BL $\quad(0.083,0.077)$ & $(0.083,0.078)$ \\
BR & $(0.864,0.112)$ & $(0.864,0.112)$ \\
\hline \hline \multicolumn{2}{c}{ Re $=5000:$} \\
TL $\quad(0.061,0.909)$ & $(0.064,0.909)$ \\
BL & $(0.073,0.138)$ & $(0.073,0.137)$ \\
BR & $(0.801,0.074)$ & $(0.806,0.073)$ \\
\hline
\end{tabular}

those reported by Erturk [Erturk, 2009]. The deviation in the $x$ and $y$ coordinate of the center of the vortex is less than $6 \%$. This deviation could be consequence of the time marching scheme used in the solver, because the vortices of the corners take a long time to reach steady state. The tolerance value used as convergence criterion must be smaller than the intensity of the corner vortices. Otherwise, these vortices may not appear in the solution.

Figure 5 shows the horizontal component of velocity as a function of time at three points within the calculation domain, named $P_{1}, P_{2}$ and $P_{3}$ and located at $(x, y)$ coordinates equal to $(L / 4, L / 4),(L / 8, L / 8)$ and $(L / 16, L / 16)$, respectively (see figure 3$)$. It can be seen the transient response in beginning of the calculation and the reaching of a steady state for $R e=1000$ and 5000 . In the case of $R e=10000$, a steady state is never reached and the velocity varies periodically, with variations in the oscillation amplitude as well. The nonstationary nature of the flow is more perceptible near the corners, where vortices emerge more markedly, con-
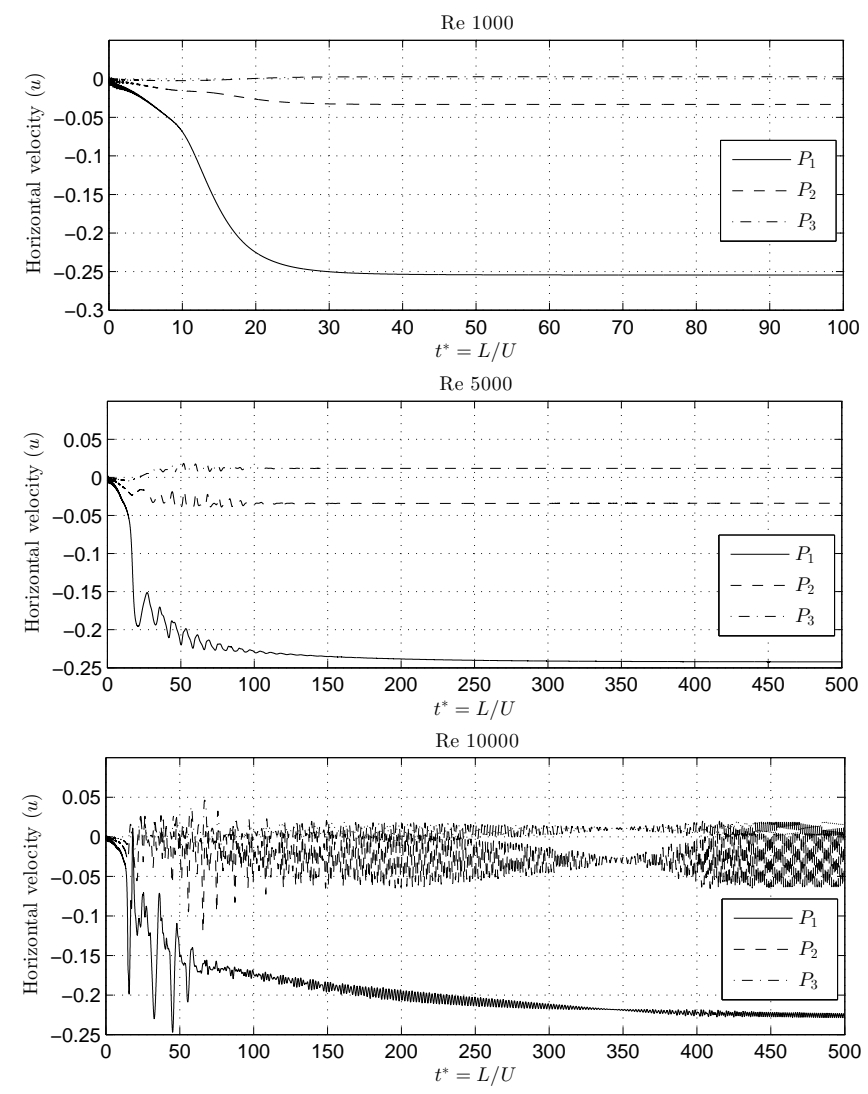

Fig. 5 Temporal evolution of the horizontal component of velocity for Reynolds numbers of 1000,5000 and 10000 at the points $P_{1}, P_{2}$ and $P_{3}$ in the calculation domain (see figure 3 ).

sequence of the higher velocity gradients. The streamlines for $\mathrm{Re}=10000$ plotted in figure 3 were calculated using a mean velocity, obtained from one hundred in- 
dividual $u$ and $v$ fields. This mean velocity field fits closely that achieved by stream-vorticy formulations.

An interesting aspect of the LDCF problem is the Reynolds number at which transition to turbulent flow occurs. Erturk et al. [Erturk et al., 2005] makes a thorough review of previous results, where solutions of the stream-vorticity formulation yield the highest Re for transition. In the velocity-pressure formulation case, results widely vary depending on the combinations of spatial discretization approaches and time-marching schemes. In this study, it was found that at $R e=10000$ and above the velocity field is no longer stationary. This calculation was carried out for discretizations of $1024^{2}, 1536^{2}$ and $2048^{2}$ nodes, obtaining the same non-stationary result in all cases. Additionally, the main frequencies of the velocity oscillations in the inspected points $\left(P_{1}\right.$ to $\left.P_{3}\right)$ were the same for all discretizations, suggesting mesh independence.

An important parameter of the parallelization scheme was analyzed. The effect of the number of thread-perblock on the global performance depends of several factors related with the GPU architecture. The analysis of these factors is not easy, but an optimum value can be identified experimentally. In order to do this, the LDCF problem for $\mathrm{Re}=400$ in a domain with $1024^{2}$ nodes was solved for a increasing number of threads-per-block. Figure 6 a shows the effect of the parallelization scheme considering both single and double precision calculations. It can be seen a better performance for a value of 128, with a processing time difference between 128 and 512 of about $20 \%$. This is an important difference and it can certainly become a deciding factor. Results were similar for single and double precision. It is important to mention that the computing device (NVIDIA GTX570) was used for graphics and calculation simultaneously.

Figure $6 \mathrm{~b}$ shows the speedup obtained for domain sizes from $256^{2}$ to $4096^{2}$ nodes and $R e=400$. The analysis was carried out for single and double precision. It can be seen an important increasing in performance when the domain gets bigger, reaching a maximum for the $2048^{2}$ size. In the $256^{2}$ case, the speedup is small because the transference time of data to and from GPU is large compared to the processing time in GPU. In the $4096^{2}$ case, the size of the matrices causes a high transference time. The maximum value is, approximately, seventy times for double precision and ninety times for single precision. This is an important increasing in the computing performance with a great effect on the processing time. For example, a 24 hours simulation could be reduced to 20 minutes.

\section{Conclusions}

In this work, a finite difference based solver for the 2D incompressible Navier-Stokes equations was programmed in both CPU and GPU. The solver allowed the solution of the Lid Driven Cavity Flow problem, providing results in agreement with literature for values of the Reynolds number up to 10000 using discretizations of $1024^{2}$ and $2048^{2}$ nodes.

The solution reached steady state for Reynolds number up to 5000, and an unsteady solution was obtained for $R e=10000$. This unsteady solution is composed of a fluctuating velocity field, with oscillations of higher amplitude near the walls and in the eddies of the corners of the cavity, suggesting a Reynolds number for turbulence transition between 5000 and 10000 for the two-dimensional case of the LDCF problem. However, as turbulence is a three-dimensional phenomenon, the real value for transition could be different from that obtained in the two-dimensional case.

Results for $\mathrm{Re}=10000$ were very similar when calculated with different discretizations. This results suggest mesh independence. Then, the fluctuations and eddies observed can be product of the solution of the NavierStokes equations and not an effect of the numerical approach employed.

A comparison between the CPU and GPU times made possible the performance analysis, showing an important increase in the computing speed using the GPU. Speedups of seventy and ninety times for double and single precision, respectively, show the advantage of GPU technology in the solution of engineering problems that require intensive numerical calculations. An analysis of the threads-per-block value, probably the most important parallelization parameter in CUDA, shows the existence of an optimum value. This result is a simple but powerful method for CUDA optimization with an important effect upon the global processing time.

A relatively low cost GPU can accelerate CFD calculations significantly. Mass production of GPUs has reduced the cost for the final consumer, and the research and innovation by manufacturers have drastically increased the computational power and improved the use of energy. An important feature is scalability, because it is possible to incorporate several devices to increase the computational power. Additionally, the software is in constant improvement, including new capabilities in the language as well as libraries for specific tasks. Then, GPU technology is an interesting and affordable option in order to accelerate engineering calculations. 


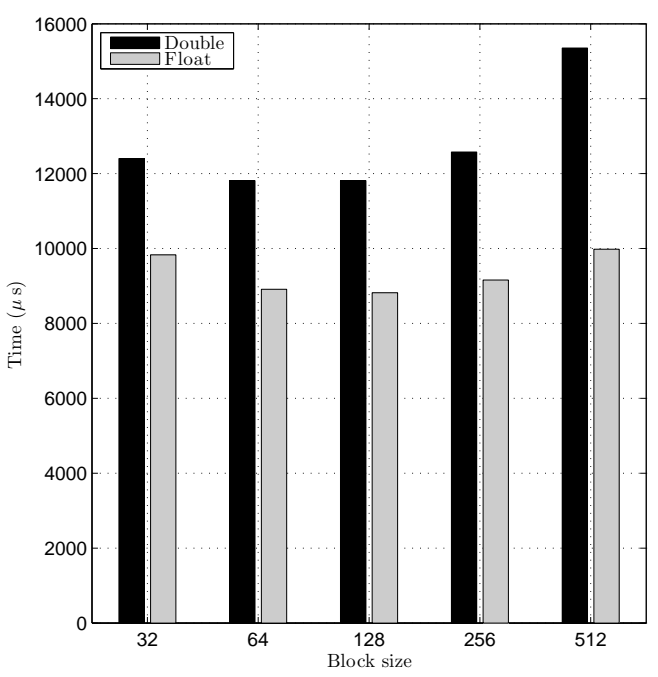

(a)

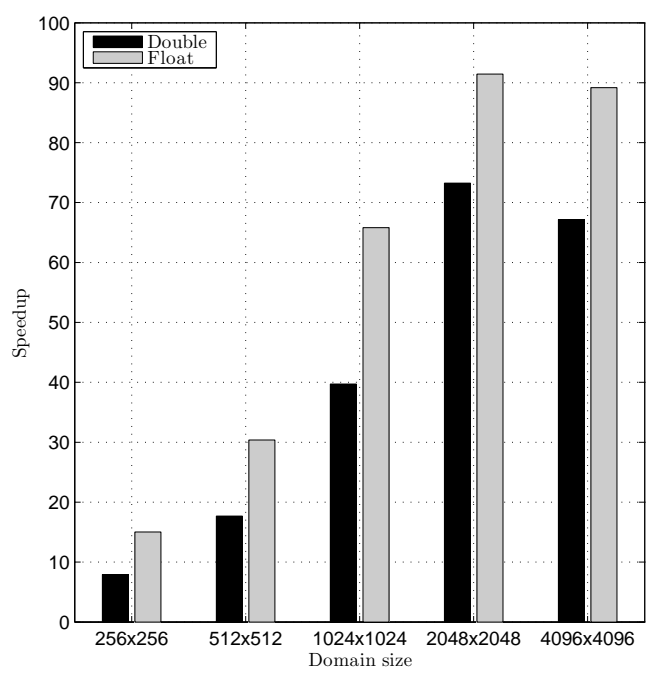

(b)

Fig. 6 Processing time obtained with the GPU (a) as a function of the block size (threads per block) and (b) speedup obtained as a function of the number of nodes in the domain using single and double precision arithmetic.

\section{Acknowledgments}

This research was supported by the Research and Technological Development Program of Universidad Autónoma de Occidente by the grant INTER11-147.

\section{References}

[FER, 2009] 2009. NVIDIA's Next Generation CUDA Compute Architecture: Fermi. Technical Report V1.1, NVIDIA Corporation.

[Bell \& Garland, 2008] Bell, N., \& M. Garland 2008. Efficient Sparse Matrix-Vector Multiplication on CUDA. Technical Report NVR-2008-004, NVIDIA Corporation.

[Cardoso \& Bicudo, 2009] Cardoso, N., \& P. Bicudo 2009. Time dependent simulation of the Lid Driven Cavity at high Reynolds number. arXiv:0809.3098 [physics.flu-dyn].

[Cecka et al., 2000] Cecka, C., A. J. Lew, \& E. darve 2000. Assembly of Finite Element Methods on Graphic Processors. International Journal for Numerical Methods in Engineering.

[Chapra \& Canale, 1995] Chapra, S. C., \& R. P Canale 1995. Métodos numéricos para ingenieros. McGrawHill/Interamericana Editores S.A. de C.V., Mexico, 6th edition.

[Chorin, 1968] Chorin, Alexandre Joel 1968. Numerical solution of the Navier-Stokes equations. Mathematics of Computation, 22:745-762.

[Courtecuisse \& Alard, ] Courtecuisse, H., \& J. Alard. Parallel Dense Gauss-Seidel Algorithm on Many-Core Processors. In Proceedings of the 11th IEEE International Conference on High Performance Computing and Comunications.

[Erturk, 2009] Erturk, E. 2009. Discussions on driven cavity flow. International Journal for Numerical Methods in Fluids, 60:275-294.

[Erturk et al., 2005] Erturk, E., T. C. Corke, \& C. Gökçöl 2005. Numerical solution of 2-D steady incompressible driven cavity flow at high Reynolds number. International Journal for Numerical Methods in Fluids, 48:747-774.

[Frezzotti et al., 2011] Frezzotti, A., G. P. Ghiroldi, \& L. Gibelli 2011. Solving model kinetic equations on GPUs. Computers \& Fluids, 50:136-146.

[Ghia et al., 1982] Ghia, U., K. N. Ghia, \& C. T. Shin 1982. High-Re Solutions for Incompressible Flow Using the Navier-Stokes Equations and a Multigrid Method. Journal of Computational Physics, 48:387-411.

[Markall et al., 2013] Markall, G. R., A. Slemmer, D. A. Ham, P. H. J. Kelly, C. D. Cantwell, \& S. J. Sherwin 2013. Finite element assembly strategies on multi-core and many-core architectures. International Journal for Numerical Methods in Fluids, 71(1):80-97.

[Moreland \& Angel, 2003] Moreland, K., \& E. Angel 2003. The FFT on a GPU. In Proceedings of the ACM SIGGRAPH/EUROGRAPHICS conference on Graphics hardware, HWWS '03, pages 112-119, Aire-la-Ville, Switzerland, Switzerland. Eurographics Association.

[Patankar, 1980] Patankar, S. V. 1980. Numerical Heat Transfer and Fluid Flow. Mc Graw Hill, USA.

[Seibold, 2008] Seibold, B. 2008. A compact and fast Matlab code solving the incompressible Navier-Stokes equations on rectangular domains (mit18086 navierstokes.m). Massachusetts Institute of Technology.

[Vanka, 2013] Vanka, S. P. 2013. 2012 Freeman Scholar Lecture: Computational Fluid Dynamics on Graphics Processing Units. Journal of Fluids Engineering, 135(6):23.

[Versteeg \& Malalasekera, 1995] Versteeg, H. K., \& W. Malalasekera 1995. An introduction to computational fluid dynamics. Addyson Wesley Longman Limited, Essex, England.

[Zheng et al., 2013] Zheng, L., H. Zhang, T. Gerya, M. Knepley, D. A. Yuen, \& Y. Shi 2013. Implementation of a multigrid solver on a GPU for Stokes equations with strongly variable viscosity based on Matlab and CUDA. International Journal of High Performance Computing Applications, 28(1):50-60. 